\title{
Beta-blockers Associated with a Mortality Benefit in Patients with Systolic Dysfunction and Elevated Serum Bilirubin
}

\author{
Christopher Labos*, Vivian Nguyen, Nadia Giannetti and Thao Huynh
}

Division of Epidemiology, Biostatistics and Occupational Health, McGill University

\begin{abstract}
Background: Hyperbilirubinemia is associated with increased mortality in heart failure (HF) patients. We evaluated the impact of evidence-based medical therapy, in particular beta-blocker on the survival of patients with $\mathrm{HF}$ and hyperbilirubinemia.

Methods and Results: We reviewed the charts of all patients followed at our tertiary care heart failure clinic. Hyperbilirubinemia was defined as total bilirubin $>30 \mu \mathrm{mol} / \mathrm{L}$ (1.5 times the upper limit of our laboratory value). The primary endpoint was all-cause mortality. The secondary endpoint was a composite of death, cardiac transplant or ventricular assistance device implantation (VAD). Of $1035 \mathrm{HF}$ patients, 121 patients $(11.7 \%)$ had hyperbilirubinemia. Median follow-up was 556 days. Hyperbilirubinemia was associated with an eight-fold increase in all-cause mortality, hazard ratio (HR): 8.78[95\% Confidence Intervals (CI): 5.89-13.06]. Beta-blocker use was associated with approximately $60 \%$ reduction in all-cause mortality (HR: $0.38,95 \% \mathrm{CI}: 0.15-0.94$ ) and 70\% reduction in the composite secondary endpoint (HR:0.31, 95\% CI:0.13-0.71) in patients with hyperbilirubinemia.
\end{abstract}

Conclusion: HF patients with hyperbilirubinemia have increased early mortality, need for cardiac transplantation or VAD. Beta-blocker use was associated with early survival benefit in these patients. Bilirubin levels should be monitored in patients with HF and early initiation of beta-blockers in patients with hyperbilirubinemia should be considered.

Keywords: Beta-blockers, bilirubin, heart Failure, prognosis

\section{INTRODUCTION}

In 1930, Joliffe [1] first reported hyperbilirubinemia in patients with heart failure (HF). The prevalence of asymptomatic hyperbilirubinemia was as high as $70 \%$ in some patient series with HF [2-4]. Although frequently asymptomatic, these laboratory abnormalities had important prognostic implications. Hyperbilirubinemia was associated with increased likelihood of inotropic requirement in patients with acutely decompensated HF [5]. Admission bilirubin levels were associated with mortality and re-admission due to HF [6]. Hyperbilirubinemia was also an independent mortality predictor following insertion of a left ventricular assist device (LVAD) [7]. Allen et al. demonstrated that hyperbilirubinemia was independently associated with allcause mortality in a large cohort of patients enrolled in the CHARM study (Candesartan in Heart failure Assessment of Reduction in Mortality and morbidity program) [8].

However, it remains unclear whether conventional HF medical treatment may lower the increased mortality associated with hyperbilirubinemia in patients with HF. Therefore, we evaluated the impact of conventional HF

\footnotetext{
*Address correspondence to this author at the Division of Cardiology, McGill University Health Center, 687 Pine Avenue West, Room M4.76, Montreal, Quebec, Canada, H3A 1A1; Tel: 514-934-1934;

Fax: 514-843-2813; E-mail: christopher.labos@mail.mcgill.ca
}

medical therapy on the increased mortality associated with hyperbilirubinemia in patients with HF.

\section{METHODS}

We reviewed the charts of all patients followed at a tertiary care heart failure clinic (during the years 2000-2009). We included all patients with more than one HF clinic visit. We excluded patients without recorded bilirubin levels $(\mathrm{n}=481)$ and patients with documented liver disease due to conditions other than HF $(n=12)$. Patients with HF and preserved LV systolic function define that e.g. by left ventricular ejection (LVEF) were included in this analysis $(n=260)$.

We defined hyperbilirubinemia as $>30 \mu \mathrm{mol} / \mathrm{L}$ (i.e. $>1.5$ times the upper limit of normal of our laboratory value). For the purpose of this analysis, we used New York Heart Association (NYHA) functional class at the first HF Clinic visit. Left ventricular ejection (LVEF) value was obtained from the echocardiogram closest to enrolment in the HF Clinic. Use of medication was defined as prescription of the specific medication at time during follow-up.

The primary end-point was all cause mortality and the secondary end-point was a composite end-point of mortality, or cardiac transplantation or implantation of VAD. For patients with more than one secondary endpoint, only the first occurring event was taken into account. Events were 
censored at the time of the event or at the last clinic visit if the patient did not have any endpoint.

\section{Statistical Analysis}

Comparison of categorical variables was carried out by chi-square testing and continuous variables were compared with Student t-test (values with normal distributions) or nonparametric Kruskal-Wallis testing (values with nonparametric distributions). Differences between groups were considered as significant when $p<0.05$. Univariate analyses were performed to identify covariates potentially associated with all-cause mortality and with the secondary endpoints. For identification of independent predictors of primary and secondary endpoints, only clinical characteristics with potential associations $(\mathrm{p}<0.10)$ were entered in the models. For evaluation of impact of medical therapy, we also entered types of medication commonly used in patients with heart disease such as beta-blockers, angiotensin converting enzyme inhibitors (ACEI), angiotensin receptor blockers (ARB), digitalis, statins and calcium channel blockers (CCB) in the models.

Survival analyses were performed with Cox proportional hazards models. All analyses were carried out using IBM SPSS 20.0 software.

\section{RESULTS}

There were 1035 patients with available bilirubin levels and 121 patients $(11.7 \%)$ had hyperbilirubinemia. Patients with hyperbilirubinemia had lower use of beta-blockers, statins and calcium-blocker, but more spironolactone use and lower mean initial NYHA class than patients with normal bilirubinemia (Table 1). These patients also had a higher rate of implantation of biventricular implantable cardioverter defibrillator (BIV-ICD) $(8 \quad v s .4 \%)$. Other baseline characteristics were similar between patients with hyperbilirubinemia and normal bilirubin levels (Table 1). The median follow-up was 556 days (Q1,Q3:156,1335). There were 108 deaths, (10.4\%), with 50 deaths $(5.5 \%)$ in patients with normal bilirubinemia and $58(47.9 \%)$ in patients with hyperbilirubinemia. In patients with normal bilirubinemia, 5 patients $(0.5 \%)$ had VAD implantation and $17(1.9 \%)$ underwent cardiac transplantation. Nine patients (7.4\%) with hyperbilirubinemia had VAD implantation and 3 $(2.5 \%)$ underwent cardiac transplantation. In total, 123 patients $(11.8 \%)$ had one of the three above endpoints; 63 $(6.9 \%)$ of patients with normal bilirubinemia and $60(49.6 \%)$ of the patients with hyperbilirubinemia (Table 2 ).

Independent predictors for all-cause mortality and secondary endpoints are shown in Table 3.

Table 1. Baseline characteristics of patients

\begin{tabular}{|c|c|c|c|c|}
\hline & All n=1035 & Normal Bilirubinemia $n=914$ & Hyperbilirubinemia $n=121$ & p-values \\
\hline Age (years), mean (SD) & $64.3(15.7)$ & $64.5(15.5)$ & $62.9(16.7)$ & NS \\
\hline Females $(\%)$ & 29.0 & 29.8 & 23.3 & NS \\
\hline Diabetes mellitus (\%) & 32.3 & 33.2 & 25.6 & NS \\
\hline Atrial fibrillation (\%) & 22.9 & 22.5 & 39.3 & $<0.001$ \\
\hline Ischemic cardiomyopathy (\%) & 44.7 & 45.3 & 40.5 & NS \\
\hline LVEF (\%), mean (SD) & $29.6(16.4)$ & $29.8(16.3)$ & $28.3(17.0)$ & NS \\
\hline NYHA class, mean (SD) & $2.1(0.8)$ & $2.3(0.8)$ & $2.0(0.8)$ & $<0.001$ \\
\hline Implantable defibrillator (\%) & 21.9 & 22.0 & 21.5 & NS \\
\hline Biventricular defibrillator(\%) & 4.7 & 4.2 & 8.6 & 0.04 \\
\hline Creatinine $(\mu \mathrm{mol} / \mathrm{L})$, median $(\mathrm{Q} 1, \mathrm{Q} 3)$ & $96.5(80.0,123.7)$ & $104.0(86.5,131.0)$ & $96.0(79.0,122.0)$ & NS \\
\hline Beta-blocker $(\%)$ & 89.0 & 89.9 & 81.8 & 0.05 \\
\hline ACEI $(\%)$ & 78.1 & 78.4 & 76.8 & NS \\
\hline $\mathrm{ARB}(\%)$ & 35.5 & 35.0 & 39.7 & NS \\
\hline Digoxin $(\%)$ & 50.9 & 49.0 & 65.3 & $<0.001$ \\
\hline Statin $(\%)$ & 68.1 & 69.2 & 59.5 & 0.03 \\
\hline Calcium blocker (\%) & 27.8 & 29.0 & 19.0 & 0.03 \\
\hline Spirinolactone $(\%)$ & 27.4 & 25.0 & 45.1 & $<0.001$ \\
\hline
\end{tabular}

ACEI: Angiotension converting enzyme inhibitor

ARB: Angiotensin receptor blocker

LVEF: Left ventricular ejection fraction

NS: Non significant

NYHA: New York Heart Association

SD: Standard deviation 
Hyperbilirubinemia was associated with an eight-fold increase in all-cause mortality and in the composite secondary endpoint. There was a $4 \%$ increase in all-cause mortality associated with every year increase in age. Every percentage increase in LVEF correlated with a two-percent reduction in mortality and every increase in NYHA class was associated with $40 \%$ increase in mortality. Presence of an ICD was associated with $50 \%$ decrease in mortality. Hyperbilirubinemia, age, LVEF, NYHA and ICD had similar impacts on the secondary endpoint. Ischemic cardiomyopathy, coronary revascularization, female sex and diabetes mellitus were not associated independently with allcause mortality and the secondary endpoint.

There were early separations of the survival curves for all-cause mortality and the secondary endpoint between patients with hyperbilirubinemia and normal bilirubinemia (Figs. 1a and 1b). At approximately 200 days during followup, there was already a $10 \%$ difference in survival free of allcause mortality and the secondary endpoint between the two groups of patients. Sensitivity analyses revealed similar results with adjustment for differences in medical therapies and exclusion of patients with preserved LVEF.

We further examined the impact of conventional HF medications on all-cause mortality, stratified for hyperbilirubinemia and adjusted for baseline characteristics (such as age, gender, ischemic cardiomyopathy, NYHA, LVEF and ICD) (Table 4). In patients with hyperbilirubinemia, use of beta-blockers was independently associated with $60 \%$ reduction in all-cause mortality and a $70 \%$ reduction in the secondary endpoint (Table 5). In patients who received beta-blockers, the median times to death and the secondary endpoints were 631 days (quartile 1 , quartile 3 (Q1,Q3): 261, 1325) and 616 days ((Q1,Q3):104,924), respectively. In patients who did not receive beta-blockers, these times were $540(\mathrm{Q} 1, \mathrm{Q} 3: 26,951)$ and 492 days (Q1,Q3:25,847), respectively. (Fig. 2a).

In patients with normal bilirubinemia, the survival benefit of beta-blockers was inconclusive (Table $\mathbf{4}$ and Fig. 2b). In these patients, ACEI, ARB and statins were independently associated with reductions in all-cause mortality and digoxin was associated with 3.5 times increase in all-cause mortality and two-fold increases in secondary endpoint. Spironolactone and calcium channel blockers were not associated with all cause mortality or the composite endpoint.

\section{DISCUSSION}

We showed that hyperbilirubinemia was frequent in HF patients and was independently associated with increased allcause mortality and need for cardiac transplantation or VAD.

Table 2. Primary and secondary endpoints.

\begin{tabular}{|l|c|c|c|}
\hline & All $\mathbf{n = 1 0 3 5}$ & Normal Bilirubinemia n=914 & Hyperbilirubinemia n=121 \\
\hline \hline All-cause mortality, $\mathrm{n}(\%)$ & $108(10.0)$ & $50(5.5)$ & $58(47.9)$ \\
\hline VAD, $\mathrm{n}(\%)$ & $14(1.4)$ & $5(0.5)$ & $9(7.4)$ \\
\hline Transplant, $\mathrm{n}(\%)$ & $20(2.0 \%)$ & $17(1.9 \%)$ & $3(2.5 \%)$ \\
\hline Death, VAD or Transplant, $\mathrm{n}(\%)$ & $123(11.8 \%)$ & $63(6.9 \%)$ & $60(49.6 \%)$ \\
\hline
\end{tabular}

VAD: Ventricular assist device
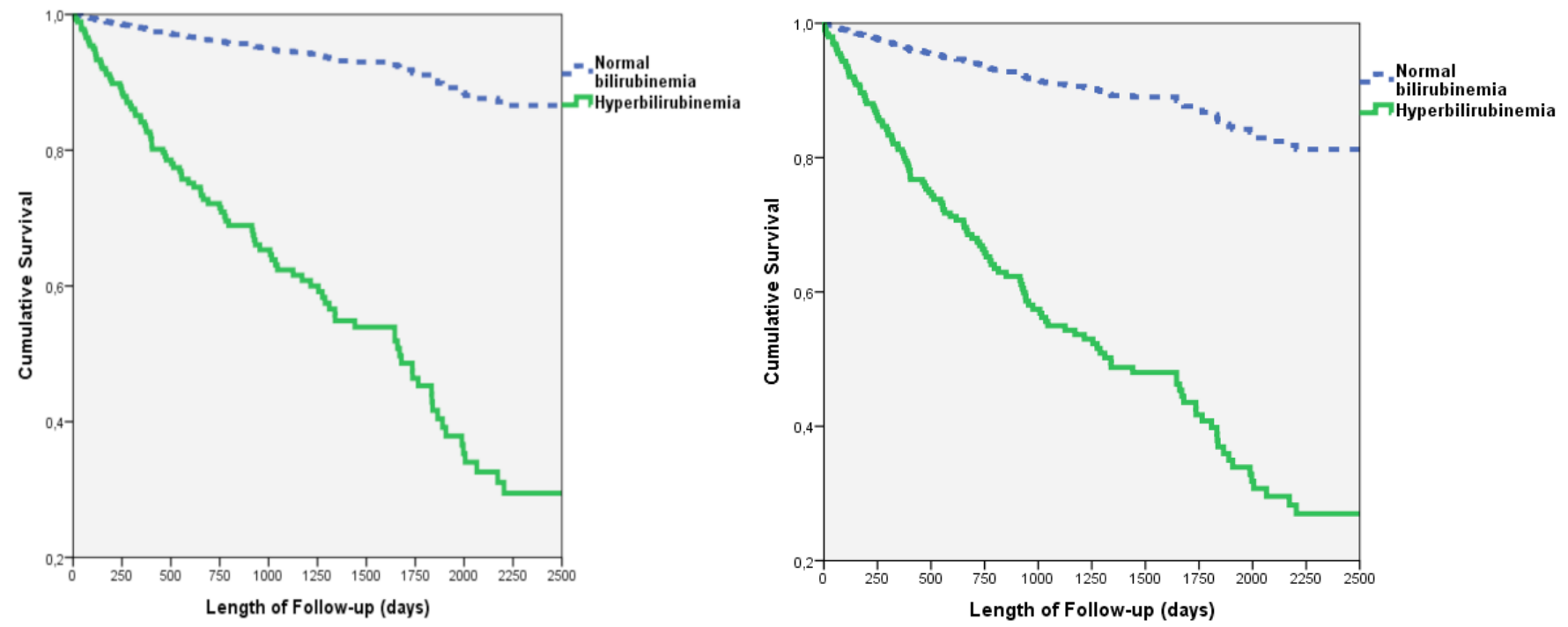

Fig. (1). (a). All-cause mortality in patients with heart failure stratified by bilirubinemia, (b). All-cause mortality or cardiac transplantation or ventricular assist device in patients with heart failure stratified by bilirubinemia. 
Table 3. Independent predictors of primary and secondary endpoints.

\begin{tabular}{|l|c|c|c|c|}
\hline \multirow{2}{*}{ Predictors } & \multicolumn{2}{|c|}{ All-cause Mortality } & All-cause Mortality, Cardiac Transplantation or Ventricular assist Device \\
\cline { 2 - 5 } & HR (95\% CI) & P-values & HR (95\% CI) & P-values \\
\hline \hline Hyperbilirubinemia & $8.78(5.89-13.06)$ & $<0.001$ & $8.26(5.76-11.84)$ & $<0.001$ \\
\hline Age & $1.04(1.02-1.05)$ & $<0.001$ & $1.02(1.01-1.03)$ & 0.01 \\
\hline LVEF & $0.98(0.97-0.99)$ & 0.02 & $0.98(0.97-0.99)$ & 0.006 \\
\hline NYHA & $1.41(1.05-1.89)$ & 0.02 & $1.46(1.12-1.90)$ & 0.005 \\
\hline ICD & $0.50(0.30-0.84)$ & 0.008 & $0.37(0.23-0.60)$ & 0.001 \\
\hline
\end{tabular}

CI: Confidence Interval

ICD: Implantable cardioverter defibrillator

LVEF: Left ventricular ejection fraction

NYHA: New York Heart Association

Table 4. Impact of medical therapy on all-cause mortality stratified by bilirubin levels.

\begin{tabular}{|c|c|c|c|c|}
\hline \multirow{2}{*}{ Medications } & \multicolumn{4}{|c|}{ All-cause Mortality } \\
\hline & HR $(95 \%$ CI $)$ & p-values & HR $(95 \% \mathrm{CI})$ & p-values \\
\hline Beta-Blocker & $0.38(0.15-0.94)$ & 0.04 & $0.69(0.29-1.67)$ & 0.41 \\
\hline ACEI & $0.67(0.25-1.79)$ & 0.42 & $0.31(0.13-0.74)$ & 0.008 \\
\hline Digoxin & $0.74(0.37-1.49)$ & 0.40 & $3.50(1.53-7.96)$ & 0.003 \\
\hline Statin & $0.60(0.30-1.23)$ & 0.17 & $0.33(0.16-0.66)$ & 0.002 \\
\hline Calcium channel blockers & $0.78(0.42-1.67)$ & 0.78 & $0.91(0.54-1.54)$ & 0.74 \\
\hline Spirinolactone & $1.48(0.82-2.66)$ & 0.20 & $0.85(0.49-1.45)$ & 0.54 \\
\hline
\end{tabular}

ACEI: Angiotension converting enzyme inhibitor

ARB:Angiotensin receptor blocker

These increases in all-cause mortality and the secondary endpoint occurred early in patients with hyperbilirubinemia. Beta-blocker use was independently associated with improved early survival in patients with hyperbilirubinemia while the survival benefit of beta-blockers was less conclusive in HF patients with normal bilirubinemia.

Allen et al. reported a prevalence of $13 \%$ of hyperbilirubinemia in HF patients enrolled in the CHARM study [8]. Hyperbilirubinemia was defined as serum total bilirubin $>17 \mu \mathrm{mol} / \mathrm{L}$ by these authors. In our cohort, $35 \%$ of patients had total bilirubin greater than $17 \mu \mathrm{mol} / \mathrm{L}$ and $28 \%$ of patients had total bilirubin greater than the normal value of our laboratory $(20 \mu \mathrm{mol} / \mathrm{L})$. The prevalence of hyperbilirubinemia (defined as total bilirubin $>30 \mu \mathrm{mol} / \mathrm{L}$ ) was $12 \%$ in our stable HF patients.

Hyperbilirubinemia may be secondary to elevated rightsided venous pressure and right ventricular (RV) dysfunction. RV volume overload can result in hepatic congestion and dysfunction [3-6]. RV dysfunction is a wellestablished marker of increased mortality risk in HF patients [9-12]. Total bilirubin has been incorporated in a risk score to predict $\mathrm{RV}$ dysfunction following implantation of a LVAD [13].
Patients with elevated right-sided venous pressure and/or RV dysfunction are generally severely symptomatic with decreased exercise capacity and higher NYHA class. [14-16] Although we did not record clinical or hemodynamic measurements of elevated right-sided venous pressure or RV dysfunction, our patients with hyperbilirubinemia had lower initial NYHA functional classes $(5.6 \%$ in patients with NHYA class 1 and 5.2\% in patients with NYHA class 2) and lower mean initial NYHA functional class in our patients compared to patients with normal bilirubinemia (2.0 vs. 2.3). This fact may suggest that factors other than elevated rightsided venous pressure and/or RV dysfunction are responsible for the development of hyperbilirubinemia.

Our finding of hyperbilirubinemia as an independent predictor of all-cause mortality was in agreement with previous publications $[8,17,18]$. Hyperbilirubinemia has been shown to be associated with a $90 \%$ increase in risk of cardiovascular $(\mathrm{CV})$ death or HF hospitalization per $\mathrm{mg} / \mathrm{dl}$ increase in serum bilirubin values and a $20 \%$ increase in CV death or HF hospitalization per 1 standard deviation (SD) increase in serum levels [18]. However, these values differ from our 8-fold increased risk, possibly because we used bilirubin as a dichotomous variable with a cutoff of 30 

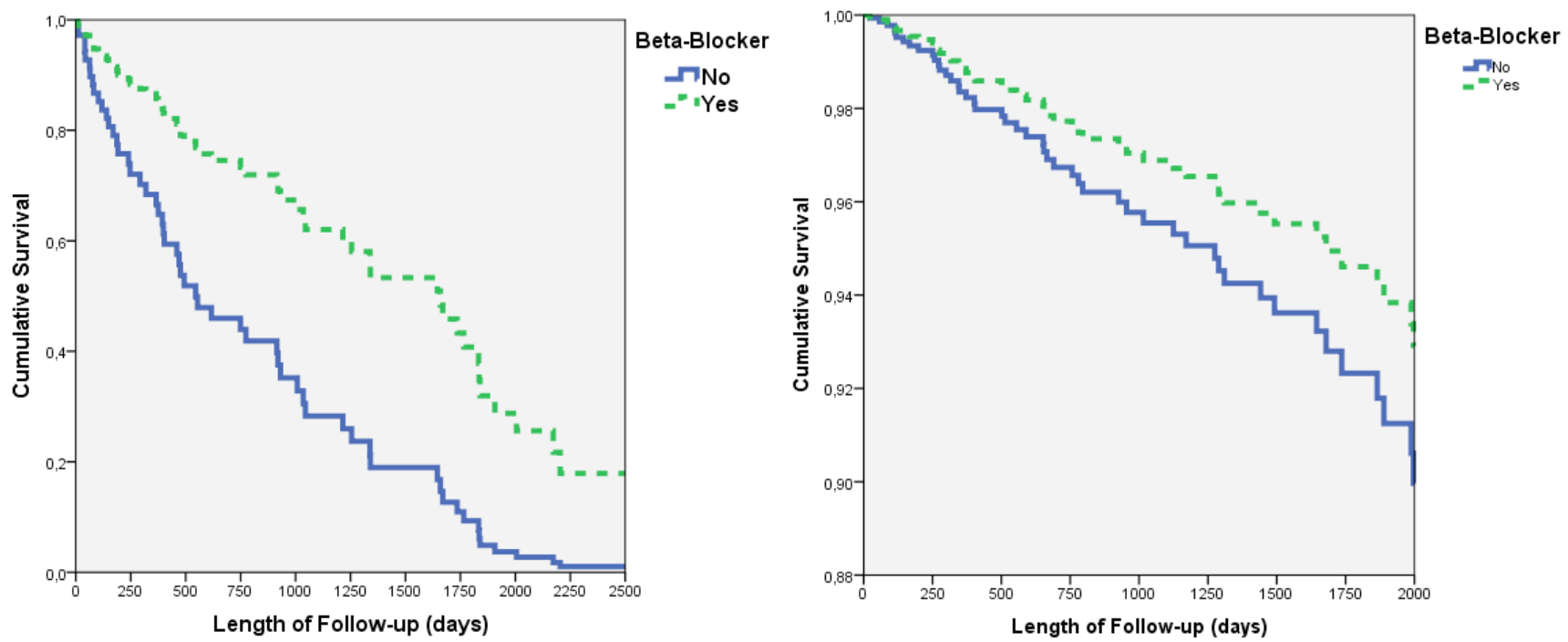

Fig. (2). (a). All-cause mortality in patients with hyperbilirubinemia, stratified by use of beta-blocker, (b). All-cause mortality in patients with normal bilirubinemia, stratified by use of beta-blockers.

Table 5. Impact of medical therapy on all-cause mortality or occurrence of cardiac transplantation or ventricular assist device stratified by bilirubin levels.

\begin{tabular}{|l|c|c|c|c|}
\hline \multirow{2}{*}{ Medications } & \multicolumn{3}{|c|}{ All-cause Mortality or Occurrence of Cardiac Transplantation or Ventricular Assistance Device } \\
\cline { 2 - 4 } & \multicolumn{2}{|c|}{ Hyperbilirubinemia } & \multicolumn{2}{c|}{ Normal Total Bilirubin Levels } \\
\cline { 2 - 5 } & HR (95\% CI) & 0.006 & \multicolumn{2}{c|}{ HR (95\% CI) } \\
\hline \hline Beta-Blocker & $0.31(0.13-0.71)$ & 0.60 & $0.39(0.19-0.80)$ & 0.009 \\
\hline ACEI & $1.27(0.51-3.14)$ & 0.14 & $0.53(0.28-1.01)$ & $0.80(0.45-1.42)$ \\
\hline ARB & $0 . .53(0.23-1.22)$ & 0.13 & $2.12(1.19-3.79)$ & 0.44 \\
\hline Digoxin & $0.61(0.32-1.17)$ & 0.11 & $0.70(0.38-1.28)$ & 0.01 \\
\hline Statin & $0.58(0.29-1.14)$ & 0.05 & $0.92(0.54-1.54)$ \\
\hline Calcium channel blockers & $0.42(0.17-1.01)$ & 0.54 & $1.60(0.98-2.63)$ \\
\hline Spirinolactone & $0.86(0.52-1.41)$ & 0.74 & 0.06 \\
\hline
\end{tabular}

ACEI: angiotension converting enzyme inhibitor

ARB:angiotensin receptor blocker

$\mu \mathrm{mol} / \mathrm{L}$ rather than a continuous variable. Our endpoints were also different from those used in most of the prior studies since we used all-cause mortality rather than $\mathrm{CV}$ death as our primary endpoint.

Furthermore, we showed that hyperbilirubinemia was independently associated with increased need of cardiac transplantation or VAD. No previous study has assessed the impact of hyperbilirubinemia on the need for transplantation or VAD insertion. The increased need of cardiac transplanttation or VAD in HF patients with hyperbilirubinemia was particularly remarkable considering that these patients already had more BIV-ICDs than HF patients with normal bilirubinemia ( 8 vs. 4\%). The early divergence of the survival curves of patients with and without hyperbilirubinemia suggested that hyperbilirubinemia may be useful to predict early mortality or need for cardiac transplantation or VAD. This finding is consistent with prior results that also showed an early divergence of the mortality curves.
Although the benefits of beta-blockers are wellestablished and are standard of care in patients with left sided HF, [19-22] their role in right-sided HF is supported only by expert opinion and limited evidence. [23,24] We showed that beta-blocker use was associated with a $60 \%$ reduction in mortality in patients with hyperbilirubinemia. The benefit of beta-blockers was evident with already a $20 \%$ difference in survival between the two treatment arms at early follow-up (200 days). The survival benefit of betablockers in patients with normal bilirubin level was less conclusive due to the low number of events in this group. In a larger sample with greater power the result may have been statistically significant.

In contrast to patients enrolled in clinical trials of betablockers, "real-life" patients may have several co-morbidities that can preclude optimal use of this medication class [25-26]. Furthermore, patients with HF of different etiologies and severities may not derive similar benefits from 
beta-blockers [26]. Therefore, a simple and easily available laboratory measure such as total bilirubin level may be a valuable guide to optimal beta-blocker use and identify patients most likely to benefit from these drugs.

The increased all-cause mortality or cardiac transplanttation or VAD with digitalis in patients with normal bilirubinemia was unexpected and may be due to chance. However, the consistency of the increased risks of digitalis for both primary and secondary endpoints in these patients supported that this finding may not be entirely fortuitous. Digitalis was associated with inconclusive reductions in primary and secondary endpoints in patients with hyperbilirubinemia. A plausible explanation would be that the sicker HF patients with hyperbilirubinemia may benefit more from the inotropic action of digitalis, while the less sick HF patients with normal bilirubinemia may gain little with inotropic reinforcement and may be more at risk of digitalis toxicity. Since we could not adjust for digoxin levels in these patients, this association cannot be fully explained. We also did not observe any survival benefit with spironolactone. However use of spironolactone was low when compared with ACE inhibitors or beta-blockers and the analysis may therefore be underpowered.

\section{LIMITATIONS}

First, due to the observational nature of our study, there were important factors that may have confounded our results. Hyperbilirubinemia may not be an independent predictor of all-cause mortality, need for cardiac transplantation or VAD but rather an indicator of more severe HF with right ventricular dysfunction and increased right-sided venous pressures. We did not assess systematically the function of the RV and could not adjust for this potential confounder. However, it is of note that patients with hyperbilirubinemia and normal total bilirubin levels had similar LVEF, and patients with hyperbilirubinemia actually had lower mean initial NYHA class. Therefore, it was less likely that the association of hyperbilirubinemia with all-cause mortality and the secondary endpoint could be entirely due to RV dysfunction.

Second, the lack of conclusive reduction in all-cause mortality of beta-blockers in patients without hyperbilirubinemia may be due to a smaller number of events. However, the significant mortality reductions associated with ACEI and ARB in patients with normal bilirubinemia suggested that our sample size should be adequately powered to detect a survival benefit with betablockers. Third, we did not assess duration or adherence to prescribed medical therapy. Therefore, we could not evaluate the relationship between the dosing of medical therapy and outcome. Finally, our cohort only included stable HF patients followed in our outpatient clinic. Thus, our findings cannot be generalized to patients with acute decompensated or hemodynamically unstable HF.

\section{CONCLUSIONS}

Hyperbilirubinemia was associated with increased early all-cause mortality, need for cardiac transplantation or VAD patients with HF. In stable HF patients, hyperbilirubinemia may identify patients at risk of early death, or cardiac transplantation or VAD who would benefit more from early initiation of beta-blockers. Future studies may clarify further the survival benefit of beta-blockers in less stable HF patients with hyperbilirubinemia. The differential impact of digitalis in HF patients with and without hyperbilirubinemia may be elucidated in larger studies.

There is evidence that bilirubin protects from vascular events. You need to mention this although it does not apply to HF. You may find the ref listed below useful as a source of literature.

Breimer LH, Mikhailidis DP. Is bilirubin a marker of vascular disease and/or cancer and is it a potential therapeutic target? Curr Pharm Des 2011; 17(33): 36443655 .

$\begin{array}{lll}\text { ABREVIATIONS } & \text { Angiotensin converting enzyme inhibitor } \\ \mathrm{ACEI} & = & \text { Angiotensin receptor blocker } \\ \mathrm{ARB} & = & \text { Beta-blocker } \\ \mathrm{BB} & = & \text { Bi-ventricular pacemaker } \\ \mathrm{BIV} & = & \text { Heart failure } \\ \mathrm{CV} & = & \text { Hazard ratio } \\ \mathrm{HF} & \text { Implantable cardioverter defibrillator } \\ \mathrm{HR} & = & \text { Left ventricular assist device } \\ \mathrm{ICD} & = & \text { Left ventricular ejection fraction } \\ \mathrm{LVAD} & = & \text { New York Heart Association } \\ \mathrm{LVEF} & = & \text { First and third quartiles } \\ \mathrm{NYHA} & = & \text { Right ventricular } \\ \mathrm{Q} 1, \mathrm{Q} 3 & = & \text { Ventricular assist device } \\ \mathrm{RV} & =\end{array}$

\section{FUNDING}

This work was not supported by any external sources of funding.

\section{CONFLICTS OF INTEREST}

The authors confirm that this article content has no conflict of interest.

\section{ACKNOWLEDGEMENTS}

Declared None.

\section{REFERENCES}

[1] Jolliffe, N. Liver function in congestive heart failure. J Clin Invest 1930; 8:419.

[2] Dunn GD, Hayes P, Breen KJ, Schenker S. The liver in congestive heart failure: a review. Am J Med Sci 1973; 265:174.

[3] Giallourakis CC, Rosenberg PM, Friedman LS. The liver in heart failure. Clin Liver Dis 2002; 6:947-67.

[4] Kubo SH, Walter BA, John DH, Clark M, Cody RJ. Liver function abnormalities in chronic heart failure. Influence of systemic hemodynamics. Arch Intern Med 1987; 147:1227-30. 
[5] Shinagawa $\mathrm{H}$, Inomata $\mathrm{T}$, Koitabashi $\mathrm{T}$, et al. Increased serum bilirubin levels coincident with heart failure decompensation indicate the need for intravenous inotropoic agents. Int Heart J 2007; 48(2):195-204.

[6] Zhang YH, Zhang J, Wang D, Lu R, Wei BQ, Kang LM. The association study of serum total bilirubin, plasma N-terminal-proBNP and invasive hemodynamic parameters in patients with heart failure. Chin J Int Med 2010; 49(4): 313-5.

[7] Shiga T, Kinugawa K, Hatano M, et al. Age and preoperative total bilirubin level can stratify prognosis after extracorporeal pulsatile left ventricular assist device implantation. Circ J 2010; 75: 121-8.

[8] Allen LA, Felker GM, Pocock S, et al. Liver function abnormalities and outcome in patients with chronic heart failure: data from the Candesartan in Heart Failure: Assessment of Reduction in Mortality and Morbidity (CHARM) program. Eur J Heart Fail 2009; 11: 170-7.

[9] Polak JF, Holman BL, Wynne J, Colucci WS. Right ventricular ejection fraction: an indicator of increased mortality in patients with congestive heart failure associated with coronary artery disease. J Am Coll Cardiol 1983; 2: 217-24.

[10] Meluzin J, Spinarova L, Hude P, et al. Prognostic importance of various echocardiographic right ventricular functional parameters in patients with symptomatic heart failure. J Am Soc Echocardiogr 2005; 18: 435-44.

[11] Sun JP, James KB, Yang XS, et al. Comparison of mortality rates and progression of left ventricular dysfunction in patients with idiopathic dilated cardiomyopathy and dilated versus nondilated right ventricular cavities. Am J Cardiol 1997; 80: 1583-7.

[12] Lewis JF, Webber JD, Sutton LL, Chesoni S, Curry CL. Discordance in degree of right and left ventricular dilation in patients with dilated cardiomyopathy: recognition and clinical implications. J Am Coll Cardiol 1993; 21:649.

[13] Matthews JC, Koelling TM, Pagani FD, Aaronson KD. The right ventricular failure risk score a pre-operative tool for assessing the risk of right ventricular failure in left ventricular assist device candidates. J Am Coll Cardiol 2008; 51:2163-2172.

[14] Di Salvo TG, Mathier M, Semigran MJ, Dec GW. Preserved right ventricular ejection fraction predicts exercise capacity and survival in advanced heart failure. J Am Coll Cardiol 1995; 25:1143-53.

[15] Baker BJ, Wilen MM, Boyd CM, Dinh H, Franciosa JA. Relation of right ventricular ejection fraction to exercise capacity in chronic left ventricular failure. Am J Cardiol 1984; 54: 596-9.
[16] Desai RV, Meyer P, Ahmed MI, et al. Relationship between left and right ventricular ejection fractions in chronic advanced systolic heart failure: insights from the BEST trial. Eur J Heart Fail 2011; 13(4): 392-7.

[17] Batin P, Wickens M, McEntegart D, Fullwood L, Cowley AJ. The importance of abnormalities of liver function tests in predicting mortality in chronic heart failure. Eur Heart J 1995; 16: 1613-8.

[18] Shinagawa $\mathrm{H}$, Inomata $\mathrm{T}$, Koitabashi $\mathrm{T}$, et al. Prognostic significance of increased serum bilirubin levels coincident with cardiac decompensation in chronic heart failure. Circ J 2008; 72: 364-9.

[19] Dickstein K, Cohen-Solal A, Filippatos G, et al. ESC Guidelines for the diagnosis and treatment of acute and chronic heart failure 2008: the Task Force for the Diagnosis and Treatment of Acute and Chronic Heart Failure 2008 of the European Society of Cardiology. Eur J Heart Fail 2008; 10(10): 933-89.

[20] Jessup. M, Abraham WT, Casey DE, et al. 2009 Focused Update: ACCF/AHA Guidelines for the Diagnosis and Management of Heart Failure in Adults. Circulation 2009; 119(14): 1977-2016.

[21] Heart Failure Society of America. HFSA 2006 Comprehensive Failure Guidelines. J Card Fail 2006; 12(1) e1-e122.

[22] Arnold JMO, Liu P, Demers C, et al. Canadian Cardiovascular Society consensus conference recommendations on heart failure 2006: Diagnosis and Management. Can J Cardiol 2006; 22(1): 23 45 .

[23] Quaife RA, Christian PE, Gilbert EM, Datz FL, Volkman K, Bristow MR. Effects of carvedilol on right ventricular function in chronic heart failure. Am J Cardiol 1998; 81:247-250.

[24] Howlett JG, McKelvie RS, Arnold JMO, et al. Canadian Cardiovascular Society Consensus Conference guidelines on heart failure, update 2009: Diagnosis and management of right-sided heart failure, myocarditis, device therapy and recent important clinical trials. Can J Cardiol 2009; 25(2): 85-105.

[25] O'Connor CM, Fiuzat M, Swedberg K, et al. Influence of Global Region on Outcomes in Heart Failure Beta-Blocker Trials. J Am Coll Cardiol 2011; 58: 915-922.

[26] Hernandez AF, Hammill BG, O'Connor CM, Schulman KA, Curtis LH, Fonarow GC. Clinical effectiveness of beta-blockers in heart failure: findings from the OPTIMIZE-HF (Organized Program to Initiate Lifesaving Treatment in Hospitalized Patients with Heart Failure) Registry. J Am Coll Cardiol 2009; 53:184-192.

(C) Christopher Labos; Licensee Bentham Open.

This is an open access article licensed under the terms of the Creative Commons Attribution Non-Commercial License (http://creativecommons.org/licenses/by-nc/3.0/) which permits unrestricted, non-commercial use, distribution and reproduction in any medium, provided the work is properly cited. 